\title{
Platelet-driven coagulopathy in COVID-19 patients: in comparison to seasonal influenza
}

\section{cases}

Jianguo Zhang ${ }^{1,2+}$, Xing Huang ${ }^{3 \dagger}$, Daoyin Ding ${ }^{4+}$ and Zhimin Tao ${ }^{1 *}$ (1)

\begin{abstract}
Background: One year into the coronavirus diseases 2019 (COVID-19) pandemic we analyzed the blood coagulopathy in severe and non-severe COVID-19 patients and linked to those of influenza patients for a comparative study.

Methods: We reported 461 COVID-19 patients and 409 seasonal influenza patients admitted at separated medical centers. With their demographic data and medical history, hematological profiles with coagulation characters were emphasized, and compared between two cohorts before and after treatment.

Results: For 870 patients included in this study, their median age was (64.0, 51.0-76.0), and among them 511 (58.7\%) were male. Hypertension, diabetes, cardiovascular diseases, and bronchitis constituted the leading comorbidities. Upon hospital admission blood test results differentiated COVID-19 patients from influenza cases, and for COVID-19 patients, leukocytosis, neutrophilia, lymphocytopenia, and thrombocytopenia were associated with disease severity and mortality. In addition, COVID-19 cohort demonstrated a prolonged prothrombin time (PT) and activated partial thromboplastin time (aPTT), increased INR, shortened thrombin time and decreased fibrinogen, compared to those in influenza cohort, leaving D-dimer levels indistinguishably high between both cohorts. Platelet hyperreactivity in COVID-19 is more evident, associated with worse hyper-inflammatory response and more refractory coagulopathy. For severe COVID-19 patients administered with anticoagulants, bleeding incidence was substantially higher than others with no anticoagulant medications.
\end{abstract}

Conclusions: Comparison of coagulation characteristics between COVID-19 and influenza infections provides an insightful view on SARS-CoV-2 pathogenesis and its coagulopathic mechanism, proposing for therapeutic improvement.

Keywords: COVID-19, SARS-CoV-2, Intensive care unit, Coagulation disorder, Influenza

\section{Background}

One year after coronavirus disease 2019 (COVID-19) surfaced, the worldwide infection toll has reached over 100 million people as the public health managements

\footnotetext{
*Correspondence: jsutao@ujs.edu.cn

†Jianguo Zhang, Xing Huang and Daoyin Ding contributed equally to this work

1 Jiangsu Province Key Laboratory of Medical Science and Laboratory Medicine, School of Medicine, Jiangsu University, Zhenjiang 212013, Jiangsu, China

Full list of author information is available at the end of the article
}

face an unprecedented challenge amid this global pandemic[1]. The disease is caused by severe acute respiratory syndrome coronavirus 2 (SARS-CoV-2), which genetically falls into the $\beta$-genus of coronavirus family $[2,3]$. Appearing a particle of $\sim 60-140 \mathrm{~nm}$ with $9-12 \mathrm{~nm}$ spikes protruding from its surface, SARS-CoV-2 showed 96.2\% genomic identity to RaTG13 detected in Rhinolophus affinis [3, 4].

SARS-CoV-2 relies on angiotensin-converting enzyme 2 (ACE2) in the host for cellular entry, and this fact mirrors the susceptibility of various organs to this

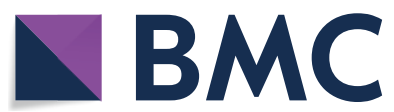

(c) The Author(s) 2021. This article is licensed under a Creative Commons Attribution 4.0 International License, which permits use, sharing, adaptation, distribution and reproduction in any medium or format, as long as you give appropriate credit to the original author(s) and the source, provide a link to the Creative Commons licence, and indicate if changes were made. The images or other third party material in this article are included in the article's Creative Commons licence, unless indicated otherwise in a credit line to the material. If material is not included in the article's Creative Commons licence and your intended use is not permitted by statutory regulation or exceeds the permitted use, you will need to obtain permission directly from the copyright holder. To view a copy of this licence, visit http://creativeco mmons.org/licenses/by/4.0/. The Creative Commons Public Domain Dedication waiver (http://creativecommons.org/publicdomain/ zero/1.0/) applies to the data made available in this article, unless otherwise stated in a credit line to the data. 
viral infection [4-6]. RNAs of SARS-CoV-2 have been detected in a variety of clinical specimens, including nasal and pharyngeal swabs, bronchoalveolar lavage fluid, sputum, blood and feces [7]. Pieced together, those findings illustrate the invasive pathway of SARS-CoV-2 into human through inhalation to cause primary lung damage, followed by secondary injuries to other organs (especially heart, liver, kidney), via either direct virus attack through blood flow or indirect insults due to systemic inflammatory response.

Studies during early COVID-19 breakout noticed heightened D-dimer levels in COVID-19 patients, especially those under critically ill conditions [8-10]. Coagulation characters in COVID-19 patients have been examined since then, where increased levels of D-dimer, fibrin degradation product, prolonged prothrombin time (PT) and activated partial thromboplastin time (aPTT) were associated with the worsened condition in COVID19 patients [11]. Anticoagulation medication using low molecular weight heparin (LMWH) lowered the 28-day mortality in COVID-19 patients with strikingly elevated D-dimer levels [12]. Concurrently, autopsy studies on deceased COVID-19 patients revealed that thrombosis was frequently observed in arteries and veins of different organs, predominantly lung and heart where viral RNA may or may not be detected [13-16]. Thus pulmonary coagulopathy contributed to the COVID-19 death, despite the usage of therapeutic anticoagulation $[14,16]$.

Coagulation disorders are associated with a diversity of viral or non-viral diseases, which depend on many factors such as the specific pathogenesis and the underlying condition of individual patient [17]. Coagulopathic characters, such as increased D-dimer concentration and von Willebrand factor activity, are also common complications in influenza infection, triggering life-threatening cytokine storm and vascular thrombosis [18]. Previously, we reported the resemblance and difference between clinical features of COVID-19 and influenza, showing that both viral infections lead to blood abnormality, including leukocytosis, neutrophilia and lymphocytopenia, although their frequencies in both illnesses could be statistically different [19]. In this study we emphasized the coagulation profiles between COVID-19 and influenza patients, with an aim to understand the hematological impact of SARS-CoV-2 infection and the potential therapeutic targets.

\section{Methods}

\section{Study participants}

This study was approved by the First People's Hospital of Jiangxia District (FPHJD) at Wuhan and the Huangshi City Hospital $(\mathrm{HCH})$ of Hubei Province, and by the Affiliated Hospital of Jiangsu University (AHJU) in Zhenjiang
City of Jiangsu Province, in China, respectively. COVID19 cases were hospitalized at FPHJD and $\mathrm{HCH}$ during January 2020 to April 2020, and influenza patients were received at AHJU during January 2018 to April 2020. For all patients, exclusion criteria were as follows: (1) patients with malignant tumors or immunodeficiency; (2) patients with genetic or acquired coagulation disorders; (3) patients with hepatic dysfunction; (4) patients on medications that may affect the blood clotting. As a result, 461 COVID-19 patients were selected, including 209 patients in the ICU and 252 patients in the non-ICU isolation ward. In parallel, 409 influenza patients were collected. For both cohorts, patient information remains anonymous, and written consent was waived by Ethics Commission of FPHJD, $\mathrm{HCH}$ and AHJU, respectively.

\section{Patient procedure}

COVID-19 patients were received at FPHJD and $\mathrm{HCH}$, diagnosed by following a standard procedure [20]. At the same time, all COVID-19 patients upon hospital admission were tested for influenza infection using IgM antibody detection against Flu A/B through indirect immunofluorescence assay (Nine respiratory pathogens IgM antibody detection kit, Zhengzhou Autobio Diagnostics Co., China). Patients included in the COVID-19 cohort of this study were all tested influenza virus $A / B$ negative. The confirmed patients were treated with antiviral drugs, including oseltamivir, arbidol, and ribavirin. For the severe patients who were admitted into the Intensive Care Unit (ICU) by following the published criteria [20], where they were receiving antibiotic treatment (sulperazone, linezolid), antifungal therapy (fluconazole, caspofungin), corticosteroid therapy, and respirationassisted ventilation. For each ICU patient, low molecule weight heparin (LMWH, 4-6 kDa) was administered 5000 IU per day via subcutaneous injection (heparin sodium for injection, manufactured by Jilin Huakang Pharmaceutical Co. Ltd., China), unless a bleeding risk was assessed. In parallel, influenza patients were diagnosed using detection kit of serum IgM antibodies against respiratory viruses based on an immunofluorescence assay (EUROIMMUN, Germany). 389 patients (95.1\%) were infected with influenza A virus (H1N1 and H3N2), 327 (80.0\%) with influenza B, and among them 307 (75.1\%) were co-infected. Influenza patients were hospitalized at $\mathrm{AHJU}$, where oxygen therapy was applied along with ribavirin or oseltamivir antiviral treatment, and none of them was transferred to ICU. For all patients, blood cell analysis was conducted by automated hematology analyzer (SYSMEX 800i, Japan; Mindray BC-5300, China), and the biochemical indicator was also analyzed (Toshiba TAB2000, Japan; Beckman AU5800, USA). Hemostatic drugs, tranexamic acid (1 g via intravenous 
infusion) or/and hemocoagulase (1 unit via intramuscular injection), were administered for patients who bled, and component blood transfusion of plasma, cryoprecipitate, or platelet was applied once necessary.

\section{Date collection and analysis}

Demographic information, medical history and blood testing results of COVID-19 and influenza patients were obtained and compared. All blood parameters were collected from patients upon hospital admission, and for blood testing after treatment, we adopted the last data of patients before their discharge from the hospital. For deceased patients, data after treatment were not collected. The categorical variables were described as frequency rates and percentages, and continuous variables were applied to describe the median and quartile range (IQR) values. Comparison of continuous variables between two groups was analyzed with Mann-Whitney test. Repeated measurements (non-normal distribution) were used following a generalized linear mixed model. $x^{2}$ test was used to compare the proportion of categorical variables, and the Fisher exact test was employed when data was limited. All statistical analyses were performed using GraphPad. A two-sided a of less than 0.05 was considered statistically significant.

\section{Results}

Comparison of baseline characteristics between COVID-19 and influenza patients upon admission

In this multicenter study, a total of 870 patients were included, of which 461 were diagnosed COVID-19 and 409 diagnosed seasonal influenza (with influenza virus A/B) upon hospital admission. Compared to the influenza cohort, the COVID-19 cohort showed a significantly shorter time interval from disease onset to hospital admission, lower median age, and the male gender ratio, but much higher mortality (Table 1). Within COVID-19 cohort, there were 252 mild patients (non-ICU group) who stayed in isolation ward until hospital discharge, and 209 severe patients later transferred to ICU for treatment (ICU group) where 90 patients unfortunately died. No statistical difference in time interval from disease onset to hospitalization was found between different groups within COVID-19. The median age of patients jumped from non-ICU, ICU survivor to ICU non-survivor, while the ratio of male gender was significantly higher in ICU group than in non-ICU group but remained indistinguishable between ICU survivors and non-survivors (Table 2). For both cohorts, hypertension, diabetes, cardiovascular diseases and bronchitis took up the primary comorbidities, echoing with our previous findings [19]. COVID-19 patients with co-existing hypertension experienced an unfavorable prognosis for disease severity and mortality, while in contrast chronic bronchitis did not contribute to disease development of COVID-19 patients (Table 2). In addition, diabetes and cardiovascular were risk factors for COVID-19 severity but not mortality.

In this study no ICU patients were admitted in the influenza cohort. Compared to influenza patients, COVID-19 patients in the non-ICU group owned much lowered age and male predisposition but similar mortality, whereas COVID-19 ICU patients had indistinguishable median age and male ratio but much elevated mortality (Additional file 1: Table S1). For both infections, cough, fever, and expectoration constituted major clinical symptoms (results not shown), where the frequency of patients undergoing fever in both cohorts was similar, but the frequency of influenza patients experiencing cough or having phlegm was higher than that of COVID-19 patients. This became in consistency with our previous report [19]. From the onset of disease when typical symptom appeared to the patient hospitalization, the time intervals in influenza cohort were prolonged compared to either non-ICU or ICU group of COVID-19 cohort.

\section{Comparison of blood parameters between COVID-19 and influenza patients before treatment}

For blood cell counts, a substantial or significant portion of patients in both cohorts demonstrated hematological abnormality, typified by leukocytosis, neutrophilia, lymphocytopenia, monocytosis, anemia and thrombocytopenia (Table 1). Compared to influenza cohort, COVID-19 cohort showed lessened severity in leukocytosis, neutrophilia and monocytosis, and increased severity in lymphocytopenia and anemia, whereas thrombocytopenia was found similar between both cohorts. Within the COVID-19 cohort, derangement in blood cell counts was generally associated with patient severity (except monocytosis) and mortality (except monocytosis and anemia) (Table 2). Using hematological data acquired upon admission, ICU patients showed worsened prognosis compared to non-ICU patients and likewise for nonsurvivors compared to survivors of ICU patients.

Notably, anemia was mirrored by low hemoglobin and low hematocrit, prevailing in patients of both cohorts ( $60 \%$ or more), and thrombocytopenia was concurrent with heightened mean platelet volume (MPV), suggesting a dearth of platelets in the bloodstream and an overdriven production of nascent platelets by megakaryocytes in the bone marrow. Within COVID-19 cohort, a poor prognosis based on anemia was closely related to the severity (but not mortality), while thrombocytopenia turned out to be a risk factor for both COVID-19 severity and mortality (Table 2). Furthermore, influenza patients showed comparable degrees of anemia 
Table 1 Baseline characteristics, blood parameters and coagulation factors in the COVID-19 cohort, compared to those in the influenza cohort

\begin{tabular}{|c|c|c|c|c|c|}
\hline & Normal & Total $(n=870)$ & COVID-19 $(n=461)$ & Influenza $(n=409)$ & $p$ value \\
\hline Onset to hospitalization, day & & $4.0(3.0-5.0)$ & $4.0(3.0-5.0)$ & $5.0(4.0-6.0)$ & $<0.0001$ \\
\hline Age & & $64.0(51.0-76.0)$ & $58.0(47.0-70.0)$ & $69.0(57.0-79.0)$ & $<0.0001$ \\
\hline Gender, male N (\%) & & $511(58.7 \%)$ & $250(54.2 \%)$ & $261(63.8 \%)$ & 0.004 \\
\hline Mortality, N (\%) & & $107(12.3 \%)$ & $98(21.3 \%)$ & $9(2.2 \%)$ & $<0.0001$ \\
\hline \multicolumn{6}{|l|}{ Comorbidity } \\
\hline Hypertension & & $281(32.3 \%)$ & $120(26.0 \%)$ & $161(39.4 \%)$ & $<0.0001$ \\
\hline Diabetes & & $97(11.1 \%)$ & $71(15.4 \%)$ & $26(6.4 \%)$ & $<0.0001$ \\
\hline Cardiovascular diseases & & $96(11.0 \%)$ & $53(11.5 \%)$ & $43(10.5 \%)$ & 0.644 \\
\hline Bronchitis & & $76(8.7 \%)$ & $34(7.4 \%)$ & $42(10.3 \%)$ & 0.131 \\
\hline \multicolumn{6}{|l|}{ Blood parameters } \\
\hline White blood cells, $\times 10^{9} / \mathrm{L}$ & $3.5-9.5$ & $6.8(5.0-9.2)$ & $6.4(4.9-8.7)$ & $7.3(5.3-9.9)$ & $<0.001$ \\
\hline$>9.5$ & & $206(23.7 \%)$ & $98(21.3 \%)$ & $108(26.4 \%)$ & 0.075 \\
\hline Neutrophils, $\times 10^{9} / \mathrm{L}$ & $1.8-6.3$ & $5.0(3.4-7.4)$ & $4.7(3.1-6.9)$ & $5.5(3.6-7.8)$ & 0.003 \\
\hline$>6.3$ & & $298(34.3 \%)$ & $143(31.0 \%)$ & 155 (37.9\%) & 0.033 \\
\hline Lymphocytes, $\times 10^{9} / \mathrm{L}$ & $1.1-3.2$ & $1.0(0.7-1.5)$ & $1.0(0.6-1.4)$ & $1.1(0.7-1.5)$ & 0.001 \\
\hline$<1.1$ & & $458(52.6 \%)$ & $270(58.6 \%)$ & $188(46.0 \%)$ & $<0.001$ \\
\hline Monocytes, $\times 10^{9} / \mathrm{L}$ & $0.1-0.6$ & $0.5(0.3-0.7)$ & $0.5(0.3-0.6)$ & $0.5(0.4-0.8)$ & $<0.0001$ \\
\hline$>0.6$ & & $263(30.2 \%)$ & $121(26.2 \%)$ & $142(34.7 \%)$ & 0.007 \\
\hline Red blood cells, $\times 10^{12} / \mathrm{L}$ & $4.3-5.8$ & $4.1(3.5-4.5)$ & $4.0(3.4-4.5)$ & $4.2(3.7-4.6)$ & $<0.001$ \\
\hline$<4.3$ & & $546(62.8 \%)$ & $300(65.1 \%)$ & $246(60.1 \%)$ & 0.133 \\
\hline Hemoglobin, g/L & $130-175$ & $123.0(106.0-136.0)$ & $120.0(100.0-135.0)$ & $126.0(111.5-137.0)$ & $<0.001$ \\
\hline$<130$ & & $541(62.2 \%)$ & $299(64.9 \%)$ & $242(59.2 \%)$ & 0.084 \\
\hline Hematocrit, \% & $40-50$ & $36.9(32.4-40.7)$ & $36.0(31.6-40.0)$ & $37.8(33.9-41.6)$ & $<0.0001$ \\
\hline$<40$ & & $611(70.2 \%)$ & $346(75.1 \%)$ & $265(64.8 \%)$ & 0.001 \\
\hline Platelet, $\times 10^{9} / \mathrm{L}$ & $125-350$ & $195.0(145.0-261.3)$ & $191.0(138.0-260.5)$ & $201.0(150.5-267.0)$ & 0.064 \\
\hline$<125$ & & $146(16.8 \%)$ & $94(20.4 \%)$ & $52(12.7 \%)$ & 0.003 \\
\hline Mean platelet volume, $\mathrm{fL}$ & $7.4-12.5$ & $10.7(9.9-11.6)$ & $10.7(10.0-11.5)$ & $10.7(9.8-11.6)$ & 0.618 \\
\hline$>12.5$ & & 85 (9.8\%) & $47(10.2 \%)$ & 38 (9.3\%) & 0.654 \\
\hline \multicolumn{6}{|l|}{ Coagulation factors } \\
\hline Prothrombin time, $s$ & $9-13$ & $12.9(11.8-14.2)$ & $13.3(12.3-14.2)$ & $12.2(11.4-14.1)$ & $<0.0001$ \\
\hline$>13$ & & $418(48.0 \%)$ & $268(58.1 \%)$ & $150(36.7 \%)$ & $<0.0001$ \\
\hline INR & $0.8-1.2$ & $1.1(1.0-1.2)$ & $1.1(1.0-1.2)$ & $1.0(1.0-1.2)$ & 0.007 \\
\hline$>1.2$ & & $179(20.5 \%)$ & $88(19.1 \%)$ & $91(22.2 \%)$ & 0.250 \\
\hline aPTT, s & $23.3-32.5$ & $29.2(26.4-32.1)$ & $30.4(28.2-32.7)$ & $27.4(24.8-31.5)$ & $<0.0001$ \\
\hline$>32.5$ & & 196 (22.5\%) & 119 (25.8\%) & 77 (18.8\%) & 0.014 \\
\hline Thrombin time, s & $14-21$ & $17.1(15.9-18.4)$ & $16.4(15.4-17.6)$ & $17.7(16.7-19.5)$ & $<0.0001$ \\
\hline$>21$ & & $109(12.5 \%)$ & $19(4.1 \%)$ & $90(22.0 \%)$ & $<0.0001$ \\
\hline Fibrinogen, g/L & $2-4$ & $4.0(3.0-5.1)$ & $3.9(2.8-4.8)$ & $4.2(3.1-5.3)$ & $<0.001$ \\
\hline$>4$ & & $429(49.3 \%)$ & $206(44.7 \%)$ & $223(54.5 \%)$ & 0.004 \\
\hline D-dimer, mg/L & $<0.55$ & $1.10(0.44-2.80)$ & $1.08(0.35-3.27)$ & $1.13(0.49-2.60)$ & 0.555 \\
\hline$>0.55$ & & $607(69.8 \%)$ & $313(67.9 \%)$ & $294(71.9 \%)$ & 0.201 \\
\hline
\end{tabular}

or thrombocytopenia to non-ICU COVID-19 patients (Additional file 1: Table S1).

Looking into coagulation factors, COVID-19 cohort demonstrated a longer PT and aPTT, higher INR, shorter thrombin time and lower fibrinogen, compared to those in influenza cohort $(p<0.05)$. However, heightened D-dimer levels were found similar between both cohorts (Table 1). Elevated D-dimer level has been reported as a prognostic indicator for COVID-19 death [11]. Our results agreed that heightened D-dimer levels had unfavored prognosis for both severity and mortality of COVID-19 infection (Table 2), and also indicated that 
Table 2 Baseline characteristics, blood parameters and coagulation factors are compared between the non-ICU and the ICU groups within COVID-19 cohort, and between the survivor and the non-survivor groups of COVID-19 ICU patients, respectively

\begin{tabular}{|c|c|c|c|c|c|c|c|}
\hline \multirow{2}{*}{$\begin{array}{l}\text { COVID-19 } \\
(n=461)\end{array}$} & \multirow[t]{2}{*}{ Normal range } & \multirow{2}{*}{$\begin{array}{l}\text { Non-ICU } \\
(n=252)\end{array}$} & \multirow{2}{*}{$\begin{array}{l}\text { ICU } \\
(\mathrm{n}=209)^{p 1}\end{array}$} & \multirow{2}{*}{$\begin{array}{l}p 1 \\
\text { value }\end{array}$} & \multicolumn{3}{|l|}{$\operatorname{ICU}(n=209)$} \\
\hline & & & & & $\begin{array}{l}\text { Survivor } \\
(n=119)\end{array}$ & $\begin{array}{l}\text { Non-survivor } \\
(\mathrm{n}=90)^{p 2}\end{array}$ & $\begin{array}{l}p 2 \\
\text { value }\end{array}$ \\
\hline $\begin{array}{l}\text { Onset to hospitaliza- } \\
\text { tion, day }\end{array}$ & & $4.0(3.0-5.0)$ & $4.0(2.0-5.0)$ & 0.254 & $4.0(3.0-5.0)$ & $3.5(2.0-5.0)$ & 0.572 \\
\hline Age & & $51.5(39.0-63.0)$ & $67.0(57.0-76.5)$ & $<0.0001$ & $63.0(55.0-76.0)$ & $69.0(58.8-78.3)$ & 0.043 \\
\hline Gender, male N (\%) & & $122(48.4 \%)$ & $128(61.2 \%)$ & 0.006 & 76 (63.9\%) & $52(57.8 \%)$ & 0.371 \\
\hline Mortality, N (\%) & & $8(3.2 \%)$ & $90(43.1 \%)$ & $<0.0001$ & $0(0)$ & $90(100 \%)$ & \\
\hline \multicolumn{8}{|l|}{ Comorbidity } \\
\hline Hypertension & & 47 (18.7\%) & $73(34.9 \%)$ & $<0.0001$ & $32(26.9 \%)$ & $41(45.6 \%)$ & 0.005 \\
\hline Diabetes & & $27(10.7 \%)$ & $44(21.1 \%)$ & 0.002 & $25(21.0 \%)$ & $19(21.1 \%)$ & 0.986 \\
\hline Cardiovascular diseases & & $8(3.2 \%)$ & $45(21.5 \%)$ & $<0.0001$ & $25(21.0 \%)$ & $20(22.2 \%)$ & 0.833 \\
\hline Bronchitis & & $17(6.7 \%)$ & $17(8.1 \%)$ & 0.570 & $6(5.0 \%)$ & $11(12.2 \%)$ & 0.060 \\
\hline \multicolumn{8}{|l|}{ Blood parameters } \\
\hline $\begin{array}{l}\text { White blood } \\
\text { cells, } \times 10^{9} / \mathrm{L}\end{array}$ & $3.5-9.5$ & $6.1(4.7-7.5)$ & $7.3(5.0-11.9)$ & $<0.0001$ & $7.0(4.9-10.1)$ & $8.6(5.5-13.0)$ & 0.015 \\
\hline$>9.5$ & & $24(9.5 \%)$ & $74(35.4 \%)$ & $<0.0001$ & $32(26.9 \%)$ & $42(46.7 \%)$ & 0.003 \\
\hline Neutrophils, $\times 10^{9} / \mathrm{L}$ & $1.8-6.3$ & $4.3(2.9-5.9)$ & $6.0(3.6-10.0)$ & $<0.0001$ & $5.1(3.4-7.8)$ & $7.3(4.2-13.0)$ & 0.001 \\
\hline$>6.3$ & & $44(17.5 \%)$ & $99(47.4 \%)$ & $<0.0001$ & $46(38.7 \%)$ & $53(58.9 \%)$ & 0.004 \\
\hline Lymphocytes, $\times 10^{9} / \mathrm{L}$ & $1.1-3.2$ & $1.1(0.8-1.6)$ & $0.8(0.5-1.1)$ & $<0.0001$ & $0.8(0.5-1.3)$ & $0.7(0.4-1.0)$ & 0.010 \\
\hline$<1.1$ & & $118(46.8 \%)$ & $152(72.7 \%)$ & $<0.0001$ & $78(65.5 \%)$ & $74(82.2 \%)$ & 0.007 \\
\hline Monocytes, $\times 10^{9} / \mathrm{L}$ & $0.1-0.6$ & $0.5(0.3-0.6)$ & $0.4(0.3-0.6)$ & 0.464 & $0.4(0.3-0.6)$ & $0.4(0.2-0.7)$ & 0.356 \\
\hline$>0.6$ & & $61(24.2 \%)$ & $60(28.7 \%)$ & 0.274 & $34(28.6 \%)$ & $26(28.9 \%)$ & 0.960 \\
\hline $\begin{array}{l}\text { Red blood } \\
\text { cells, } \times 10^{12} / \mathrm{L}\end{array}$ & $4.3-5.8$ & $4.3(3.9-4.6)$ & $3.5(2.9-4.1)$ & $<0.0001$ & $3.5(2.9-4.1)$ & $3.4(2.9-4.2)$ & 0.547 \\
\hline$<4.3$ & & $128(50.8 \%)$ & $172(82.3 \%)$ & $<0.0001$ & $102(85.7 \%)$ & $70(77.8 \%)$ & 0.137 \\
\hline Hemoglobin, g/L & $130-175$ & $128.0(115.3-140.0)$ & $107.0(86.0-124.5)$ & $<0.0001$ & $108.0(88.0-125.0)$ & $106.0(83.8-124.5)$ & 0.941 \\
\hline$<130$ & & $134(53.2 \%)$ & 165 (78.9\%) & $<0.0001$ & $94(79.0 \%)$ & $71(78.9 \%)$ & 0.986 \\
\hline Hematocrit, \% & $40-50$ & $37.8(34.6-40.9)$ & $32.2(26.7-37.2)$ & $<0.0001$ & $32.5(26.5-37.2)$ & $31.9(26.8-37.1)$ & 0.915 \\
\hline$<40$ & & $168(66.7 \%)$ & $178(85.2 \%)$ & $<0.0001$ & $103(86.6 \%)$ & 75 (83.3\%) & 0.517 \\
\hline Platelet, $\times 10^{9} / \mathrm{L}$ & $125-350$ & $201.0(152.0-260.0)$ & $173.0(106.5-269.5)$ & 0.002 & $197.0(140.0-276.0)$ & $137.5(84.8-211.3)$ & 0.001 \\
\hline$<125$ & & $27(10.7 \%)$ & $67(32.1 \%)$ & $<0.0001$ & $25(21.0 \%)$ & $42(46.7 \%)$ & $<0.0001$ \\
\hline $\begin{array}{l}\text { Mean platelet volume, } \\
f L\end{array}$ & $7.4-12.5$ & $10.5(9.7-11.2)$ & $10.9(10.3-12.1)$ & $<0.0001$ & $10.7(10.2-11.5)$ & $11.4(10.4-12.7)$ & 0.004 \\
\hline$>12.5$ & & $7(2.8 \%)$ & $40(19.1 \%)$ & $<0.0001$ & $16(13.4 \%)$ & $24(26.7 \%)$ & 0.016 \\
\hline \multicolumn{8}{|l|}{ Coagulation factors } \\
\hline Prothrombin time, $s$ & $9-13$ & $13.4(12.6-14.0)$ & $13.2(12.0-15.0)$ & 0.712 & $12.7(11.7-13.9)$ & $14.2(12.5-16.6)$ & $<0.0001$ \\
\hline$>13$ & & $157(62.3 \%)$ & $111(53.1 \%)$ & 0.046 & 50 (42.0\%) & $61(67.8 \%)$ & $<0.001$ \\
\hline INR & $0.8-1.2$ & $1.1(1.0-1.1)$ & $1.1(1.0-1.3)$ & 0.003 & $1.1(1.0-1.2)$ & $1.1(1.0-1.5)$ & 0.005 \\
\hline$>1.2$ & & $25(9.9 \%)$ & $63(30.1 \%)$ & $<0.0001$ & $27(22.7 \%)$ & $36(40.0 \%)$ & 0.007 \\
\hline aPTT, s & $23.3-32.5$ & $30.1(28.1-31.5)$ & $31.4(28.1-36.2)$ & 0.0001 & $30.7(28.0-35.3)$ & $31.7(28.6-37.7)$ & 0.224 \\
\hline$>32.5$ & & 31 (12.3\%) & $88(42.1 \%)$ & $<0.0001$ & 48 (40.3\%) & 40 (44.4\%) & 0.551 \\
\hline Thrombin time, s & $14-21$ & $15.9(15.0-17.0)$ & $17.3(16.1-18.5)$ & $<0.0001$ & $17.1(16.3-18.2)$ & $17.5(16.0-19.0)$ & 0.362 \\
\hline$>21$ & & $0(0)$ & $19(9.1 \%)$ & $<0.0001$ & $8(6.7 \%)$ & $11(12.2 \%)$ & 0.171 \\
\hline Fibrinogen, g/L & $2-4$ & $3.5(2.6-4.3)$ & $4.2(3.4-5.5)$ & $<0.0001$ & $4.2(3.4-5.4)$ & $4.3(3.3-5.5)$ & 0.831 \\
\hline$>4$ & & $85(33.7 \%)$ & $122(58.4 \%)$ & $<0.0001$ & $70(58.8 \%)$ & $51(56.7 \%)$ & 0.755 \\
\hline D-dimer, mg/L & $<0.55$ & $0.6(0.2-1.2)$ & $3.2(1.0-6.2)$ & $<0.0001$ & $2.2(0.8-5.2)$ & $3.7(1.4-7.3)$ & 0.010 \\
\hline$>0.55$ & & $134(53.2 \%)$ & $179(85.6 \%)$ & $<0.0001$ & 99 (83.2\%) & $80(88.9 \%)$ & 0.245 \\
\hline
\end{tabular}


viral infection in influenza patients induced abnormally high levels of D-dimer in analog to COVID-19. In addition, most coagulation factors showed prognostic values for COVID-19 severity but not mortality.

\section{Coagulation disorders and bleeding complications among COVID-19 and influenza patients after treatment}

For influenza patients, oxygen therapy was implemented with antiviral medications using ribavirin or oseltamivir, and none of them needed critical care, although 9 patients were unfortunately deceased in the non-ICU ward due to complications where influenza was not identified as a primary cause. After treatment, all hematological abnormalities (except anemia and prolonged PT/INR) were significantly mitigated (Table 3 ), showing a good treatability and recovery from seasonal influenza infection (Fig. 1).

For non-ICU COVID-19 patients, they were treated with broad-spectrum antibiotics including sulperazone and linezolid, and antiviral drugs including oseltamivir, arbidol and ribavirin. For COVID-19 ICU patients, they were receiving mechanical ventilation for respiratory support, while antibiotic, antiviral or/and antifungal therapeutics were also applied per necessity. Simultaneously, owing to the immobility of ICU patients, they were administered with daily LMWH unless bleeding risks were determined. As a result, many blood parameters in non-ICU COVID-19 patients were significantly ameliorated, especially fibrinogen and D-dimer levels, although discharged patients still showed common anemia (Table 4). In contrast, for ICU survivors, most hematological abnormalities did not exhibit substantial improvement upon hospital discharge, including anemia, thrombocytopenia, and most coagulopathy, although fibrinogen levels significantly dropped (Fig. 1).

Bleeding events were also observed in both cohorts during hospitalization, and major bleeding was identified to include bronchoalveolar, gastrointestinal, mucocutaneous and intracerebral hemorrhage (from high to low incidence) (Table 5). Hemostatic drugs (tranexamic acid or/and hemocoagulase) were applied to treat patients who bled, and plasma, cryoprecipitate, or platelet transfusion was applied once necessary. For COVID-19 cohort, bleeding was significantly more frequent in the ICU group than in the non-ICU group, but the bleeding occurrence was similar between survivors and non-survivors within ICU group (Additional file 1: Table S3). Consequently, tranexamic acid and component transfusion were applied more often in the ICU group when compared to the non-ICU group. Overall, influenza cohort exhibited comparable bleeding incidences at major hemorrhagic sites to COVID19 cohort, except for bronchoalveolar bleeding where COVID-19 patients had much higher frequency, and administration of hemostatic drugs or component transfusion showed similar frequency between two cohorts. However, compared to either the influenza cohort or the non-ICU COVID-19 group, the ICU group in COVID-19 cohort exhibited significantly higher bleeding occurrence, more usage of hemostatic drugs and more needs for component transfusions.

Table 3 Blood parameters and coagulation factors of influenza patients are compared before and after treatment

\begin{tabular}{|c|c|c|c|c|}
\hline Influenza & Normal range & Before treatment $(n=400)$ & After treatment $(n=400)$ & $p$ value \\
\hline \multicolumn{5}{|l|}{ Blood cell counts } \\
\hline White blood cells, $\times 10^{9} / \mathrm{L}$ & $3.5-9.5$ & $7.3(5.3-9.9)$ & $6.5(5.1-8.4)$ & $<0.0001$ \\
\hline Neutrophils, $\times 10^{9} / \mathrm{L}$ & $1.8-6.3$ & $5.4(3.6-7.7)$ & $4.8(3.4-6.6)$ & $<0.0001$ \\
\hline Lymphocytes, $\times 10^{9} / \mathrm{L}$ & $1.1-3.2$ & $1.1(0.8-1.5)$ & $1.3(0.9-1.8)$ & 0.014 \\
\hline Monocytes, $\times 10^{9} / \mathrm{L}$ & $0.1-0.6$ & $0.5(0.4-0.8)$ & $0.5(0.4-0.7)$ & 0.002 \\
\hline Red blood cells, $\times 10^{12} / \mathrm{L}$ & $4.3-5.8$ & $4.2(3.7-4.6)$ & $4.2(3.7-4.6)$ & 0.212 \\
\hline Hemoglobin, g/L & $130-175$ & $127.0(112.0-137.8)$ & $126.0(109.0-139.0)$ & 0.988 \\
\hline Hematocrit, \% & $40-50$ & $37.9(33.9-41.7)$ & $37.7(32.8-41.0)$ & 0.257 \\
\hline Platelet, $\times 10^{9} / \mathrm{L}$ & $125-350$ & $203.5(152.3-267.0)$ & $216.0(166.0-275.5)$ & $<0.0001$ \\
\hline Mean platelet volume, $\mathrm{fL}$ & $7.4-12.5$ & $10.7(9.8-11.6)$ & $10.6(10.0-11.6)$ & 0.405 \\
\hline \multicolumn{5}{|l|}{ Coagulation panel } \\
\hline Prothrombin time, $\mathrm{s}$ & $9-13$ & $12.2(11.4-14.1)$ & $11.6(10.9-12.4)$ & 0.560 \\
\hline INR & $0.8-1.2$ & $1.0(1.0-1.2)$ & $1.1(1.0-1.2)$ & 0.645 \\
\hline aPTT, s & $23.3-32.5$ & $27.4(24.7-31.3)$ & $27.3(24.9-29.7)$ & 0.027 \\
\hline Thrombin time, s & $14-21$ & $17.7(16.7-19.5)$ & $17.5(16.4-19.0)$ & $<0.0001$ \\
\hline Fibrinogen, g/L & $2-4$ & $4.2(3.2-5.3)$ & $3.5(2.8-4.1)$ & $<0.0001$ \\
\hline D-dimer, mg/L & $<0.55$ & $1.1(0.5-2.5)$ & $0.6(0.4-1.2)$ & $<0.0001$ \\
\hline
\end{tabular}



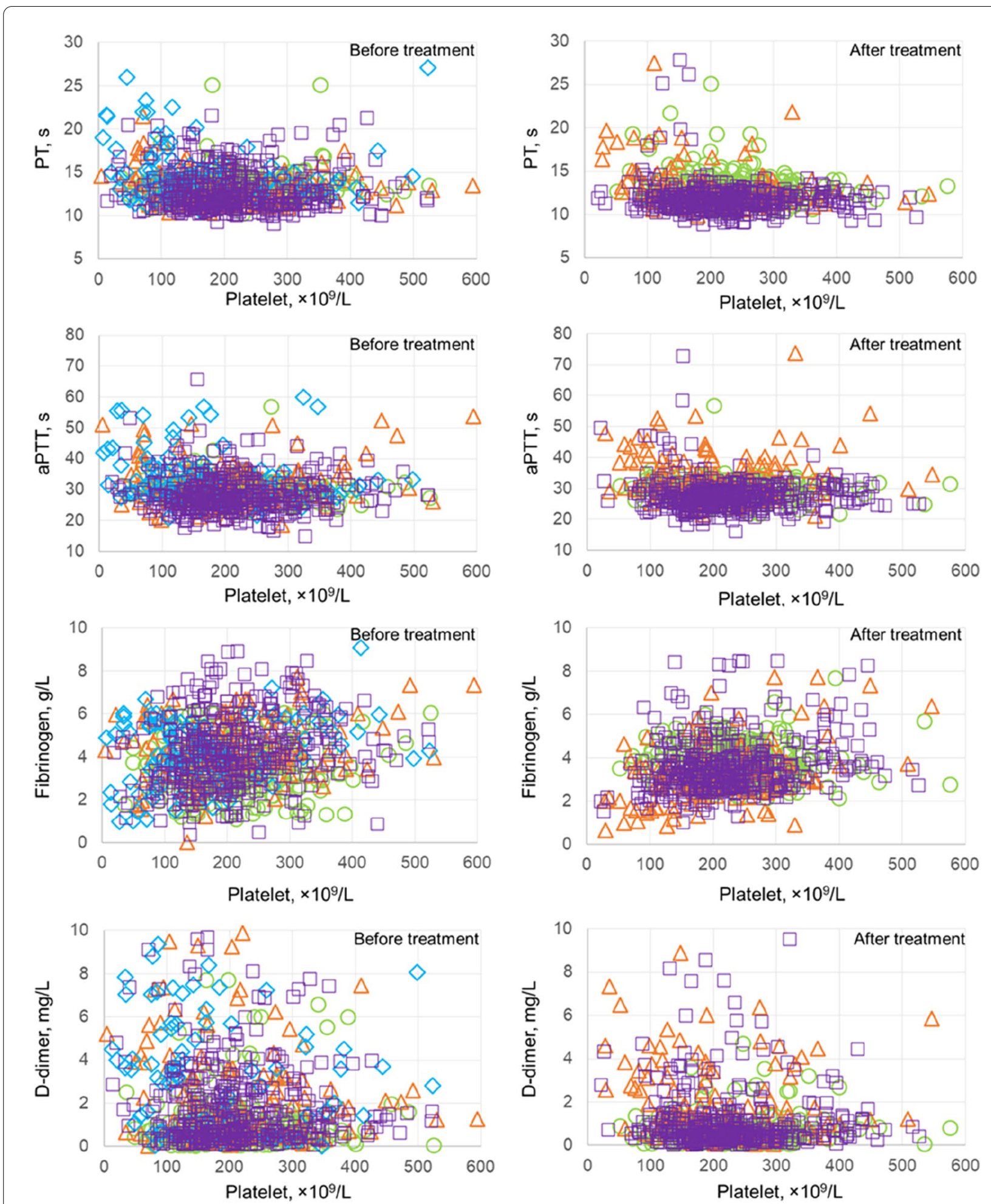

Fig. 1 PT, aPTT, fibrinogen concentration, or D-dimer level was individually plotted versus the platelet count in each patient of influenza cohort (purple square) and COVID-19 cohort (green circle for the non-ICU group, orange triangle for the ICU survivors, and blue diamond for the ICU non-survivors), before (left) and after treatment (right). Data in the ICU non-survivors were not shown after treatment 
Table 4 Blood parameters and coagulation factors of COVID-19 patients are compared before and after treatment in the non-ICU group and in the survivors of ICU group, respectively

\begin{tabular}{|c|c|c|c|c|c|c|c|}
\hline \multirow[t]{2}{*}{ COVID-19 } & \multirow[t]{2}{*}{ Normal range } & \multicolumn{3}{|l|}{ Non-ICU $(n=252)^{p 1}$} & \multicolumn{3}{|c|}{ ICU survivor $(n=119)^{p 2}$} \\
\hline & & Before treatment & After treatment & p1 & Before treatment & After treatment & p2 \\
\hline \multicolumn{8}{|l|}{ Blood cell counts } \\
\hline White blood cells, $\times 10^{9} / \mathrm{L}$ & $3.5-9.5$ & $6.1(4.7-7.5)$ & $6.3(5.3-7.4)$ & 0.019 & $7.0(4.9-10.1)$ & $7.0(5.0-9.3)$ & 0.706 \\
\hline Neutrophils, $\times 10^{9} / \mathrm{L}$ & $1.8-6.3$ & $4.3(2.9-5.9)$ & $5.0(3.8-6.1)$ & 0.001 & $5.1(3.4-7.8)$ & $4.5(2.9-6.8)$ & 0.031 \\
\hline Lymphocytes, $\times 10^{9} / \mathrm{L}$ & $1.1-3.2$ & $1.1(0.8-1.6)$ & $1.5(1.1-1.8)$ & $<0.0001$ & $0.8(0.5-1.3)$ & $1.4(1.0-1.9)$ & $<0.0001$ \\
\hline Monocytes, $\times 10^{9} / \mathrm{L}$ & $0.1-0.6$ & $0.5(0.3-0.6)$ & $0.5(0.4-0.6)$ & 0.069 & $0.4(0.3-0.6)$ & $0.5(0.4-0.7)$ & 0.008 \\
\hline Red blood cells, $\times 10^{12} / \mathrm{L}$ & $4.3-5.8$ & $4.3(3.9-4.6)$ & $4.3(4.0-4.7)$ & 0.136 & $3.5(2.9-4.1)$ & $3.5(2.8-4.2)$ & 0.569 \\
\hline Hemoglobin, $g / L$ & $130-175$ & $128.0(115.3-140.0)$ & $136.0(121.3-146.0)$ & $<0.0001$ & $108.0(88.0-125.0)$ & $97.0(81.0-121.0)$ & 0.010 \\
\hline Hematocrit, \% & $40-50$ & $37.8(34.6-40.9)$ & $39.4(36.1-42.5)$ & $<0.0001$ & $32.5(26.5-37.2)$ & $31.0(25.8-37.4)$ & 0.365 \\
\hline Platelet, $\times 10^{9} / \mathrm{L}$ & $125-350$ & $201.0(152.0-260.0)$ & $239.5(190.0-283.0)$ & $<0.0001$ & $197.0(140.0-276.0)$ & $189.0(135.0-274.0)$ & 0.567 \\
\hline Mean platelet volume, fL & $7.4-12.5$ & $10.5(9.7-11.2)$ & $10.6(10.2-11.4)$ & 0.058 & $10.7(10.2-11.5)$ & $10.6(10.0-11.7)$ & 0.074 \\
\hline \multicolumn{8}{|l|}{ Coagulation panel } \\
\hline Prothrombin time, $\mathrm{s}$ & $9-13$ & $13.4(12.6-14.0)$ & $12.7(11.8-13.7)$ & $<0.0001$ & $12.7(11.7-13.9)$ & $12.5(11.6-14.0)$ & 0.613 \\
\hline INR & $0.8-1.2$ & $1.1(1.0-1.1)$ & $1.1(1.0-1.2)$ & 0.300 & $1.1(1.0-1.2)$ & $1.1(1.0-1.2)$ & 0.007 \\
\hline aPTT, s & $23.3-32.5$ & $30.1(28.1-31.5)$ & $29.9(27.8-31.5)$ & 0.621 & $30.7(28.0-35.3)$ & $32.4(28.5-38.4)$ & 0.079 \\
\hline Thrombin time, s & $14-21$ & $15.9(15.0-17.0)$ & $15.6(14.4-16.6)$ & 0.082 & $17.1(16.3-18.2)$ & $17.5(16.3-19.4)$ & 0.343 \\
\hline Fibrinogen, g/L & $2-4$ & $3.5(2.6-4.3)$ & $3.7(3.2-4.3)$ & $<0.001$ & $4.2(3.4-5.4)$ & $3.0(2.4-3.8)$ & $<0.001$ \\
\hline D-dimer, mg/L & $<0.55$ & $0.6(0.2-1.2)$ & $0.5(0.2-0.8)$ & $<0.0001$ & $2.2(0.8-5.2)$ & $1.6(0.8-3.8)$ & 0.160 \\
\hline
\end{tabular}

Table 5 Comparison of bleeding events, usage of hemostatic drugs or component blood transfusion between the COVID-19 cohort and the influenza cohort

\begin{tabular}{|c|c|c|c|c|}
\hline & Total $(n=870)$ & COVID-19 $(n=461)$ & Influenza $(n=409)$ & $p$ value \\
\hline \multicolumn{5}{|l|}{ Bleeding events } \\
\hline Bronchus & $25(2.9 \%)$ & $21(4.6 \%)$ & $4(1.0 \%)$ & 0.002 \\
\hline Gastrointestinal tract & $24(2.8 \%)$ & $16(3.5 \%)$ & $8(2.0 \%)$ & 0.173 \\
\hline Mucocutaneous membrane & $15(1.7 \%)$ & $11(2.4 \%)$ & $4(1.0 \%)$ & 0.125 \\
\hline Intracerebral hemorrhage & $8(0.9 \%)$ & $7(1.5 \%)$ & $1(0.2 \%)$ & 0.073 \\
\hline \multicolumn{5}{|l|}{ Hemostatic drugs } \\
\hline Tranexamic acid & $37(4.3 \%)$ & $25(5.4 \%)$ & $12(2.9 \%)$ & 0.069 \\
\hline Hemocoagulase & $16(1.8 \%)$ & $11(2.4 \%)$ & $5(1.2 \%)$ & 0.219 \\
\hline Transfusion & $21(2.4 \%)$ & $15(3.3 \%)$ & $6(1.5 \%)$ & 0.087 \\
\hline
\end{tabular}

\section{Discussion}

Both SARS-CoV-2 and influenza viruses are enveloped particles that enclose viral nucleoproteins and singlestranded RNAs [19]. However, two viruses differ in many ways, such as their viral architectures, invasion patterns, and pathogenic mechanisms. With strike (S) proteins poking out on surface, SARS-CoV-2 is embedded with non-segmented positive-sense genomic RNAs (gRNAs), binding to ACE2 receptors on the host for cell entry that is facilitated by serine protease TMPRSS2 for $S$ protein priming [5]. The internalized virus releases its gRNAs into the cytoplasm, borrowing cellular ribosomes to produce polyproteins that further undertake proteolysis for activation, responsible for the next viral RNA modification, replication, translation into nascent viral components (i.e., structural proteins and gRNAs) and assembly into virions [21]. In contrast, influenza viruses employ two surface proteins for cell entry, haemagglutinin (HA) and neuraminidase (NA), by attaching to sialic acids (SAs) as host cell receptor [22]. After endocytosis, the encapsulated viral proteins and eight negative-sense RNA segments are unleashed into cytoplasm, where RNAs were relocated into nucleus for conversion into positive-stranded mRNAs, being further transported into 
cytosols for viral protein synthesis and new virion assembly before budding [23].

SA owns a broad variety on different cell types throughout the human body, while ACE2 is actively expressed along the respiratory tract, including nasal epithelium, airway, and alveolar epithelium [24-26]. Additionally, human coronavirus $\mathrm{S}$ glycoproteins possess SA-binding sites [27], and SARS-CoV-2 owns a capacity to bind to SAs [28], but with a much weaker strength than its binding to ACE2 [29]. Those findings may point out another pathogenicity of COVID-19, although more evidences are needed. Concomitantly, pre-infection by influenza viruses can augment ACE2 expression, increasing further infectivity of SARS-CoV-2 and causing more severe pulmonary damage [30]. As the flu seasons overlap with COVID-19 pandemic, preventive measures and/or timely vaccinations against both respiratory viruses are imperative.

The clinical symptoms of COVID-19 include fever, cough, dyspnea, fatigue, diarrhea and vomiting, presenting an influenza-like illness [19]. In this study, hypertension, diabetes, cardiovascular diseases and bronchitis attributed to the leading comorbidities for both infections. The median age of influenza patients was statistically higher than that of COVID-19 patients. This is in consistency with our previous finding [19], but in controversy with some of others who reported younger ages of seasonal influenza patients, especially among children $[31,32]$. The reason may lie in that young influenza patients with mild symptoms in China are reluctant to go to hospital, while suspected COVID-19 patients are obligatory to be tested and hospitalized if positive. Like high age, male gender is another risk factor for COVID19 severity and mortality, although the exact mechanism requires further investigations and opposite reports exist [33]. In general, age and gender biases have been reported among many viral diseases, and this could be associated with at least two major aspects; one contains socioeconomic traits including social and cultural traditions, living styles (e.g., smoking history) and occupational experiences, etc., and the other dedicates to biological features, exemplified by differences in age- or sex-related hormones and immune responses [33, 34].

Compared to the influenza cohort, the COVID-19 cohort showed similar frequency of leukocytosis and anemia, less frequency of neutrophilia and monocytosis, but more frequency of lymphocytopenia and thrombocytopenia. This result showed some variation from our previous observation in a different cohort study, but stood in line with others [35]. The frequencies of irregular cell counts in the patients' blood might fluctuate if small pools of data are selected. For COVID-19 patients, leukocytosis, neutrophilia, lymphocytopenia, and thrombocytopenia were associated with disease severity and mortality, being characteristic of SARSCoV-2 infection [36, 37]. Simultaneously, the prevalence of anemia was found similar between COVID-19 and influenza cohorts. After treatment, most derangements in blood cell counts were significantly alleviated in influenza and COVID-19 non-ICU patients (except anemia), but at time of hospital discharge many hematological parameters (e.g., anemia and thrombocytopenia) in COVID-19 ICU survivors did not demonstrate noticeable improvement.

Being one of common manifestations, anemia has been recognized as a prognostic indicator of COVID-19 severity but not mortality, agreeing to our finding here [38, 39]. The underlying machinery could be complexed as direct and indirect hemolysis might be causative following viral infection. Directly, SARS-CoV-2 might interplay with CD147 receptor on RBCs as another cell entry route [40], leading to rupture of erythrocytes and loss of $\mathrm{Hb}$. Indirectly, systemic inflammatory burden in COVID19 patients after lung infection may result in further prothrombotic state, including complement activation, platelet hyperreactivity, and hypoxia in major organs [41]. Consequently, erythropoiesis can be suppressed in the bone morrow, and the circulating erythrocytes can be shattered, each causing anemia. In fact, anemia is not infrequent in viral or other microbial infections, such as community-acquired pneumonia and hepatitis viruses $[42,43]$. However, in our study influenza-related anemia showed a less severity than COVID-19 one, supported by others [44]. Given the abundancy of SA on the surface of RBCs and its specificity for influenza virus binding, its direct hit on the erythrocytes can be impactful. Cytokine levels in influenza patients were reported no less than those in COVID-19 patients [45]. Therefore, both SARSCoV-2 and influenza viruses could cause anemia in a comparative pattern but to a different extent.

Thrombocytopenia has been established with a prognostic value of COVID-19 severity and mortality [11, 46]. Recent study has revealed that lung is a primary location of terminal platelet biogenesis and a major reservoir for hematopoietic progenitors [47]. Migrating from extrapulmonary sites (e.g., bone marrow), the megakaryocytes and hematopoietic progenitors reside in the lung, generating $\sim 50 \%$ of all platelets there before their release into circulation, and in case of thrombocytopenia, those progenitors displace outside the lung to re-supply platelets in the blood [47]. Rich ACE2 and TMPRSS2 expressions have been confirmed in platelets or platelet-producing megakaryocytes, suggestive of a direct viral hit [48]. Thus, the lung infection by SARS-CoV- 2 can trigger double whammy on platelets, causing increased destruction and decreased production. This viremic effect 
undermined megakaryocytes and instigated platelet activation via mitogen-activated protein kinase (MAPK) pathway $[48,49]$. Moreover, in a substantial portion of COVID-19 patients, thrombocytopenia was found together with prolonged PT/INR and aPTT, and elevated levels of fibrinogen and D-dimer, but change in thrombin time was marginal. Our results indicated a unique profile of coagulopathy, revealing a hypercoagulative and hypofibrinolytic state in the COVID-19 patients [46, 50, 51]. Consequently, platelets in COVID-19 patients tend to form aggregates with immune cells, including $\mathrm{T}$ cells, monocytes, and neutrophils, showing platelet hyperreactivity that contributes to immunothrombosis $[49,52,53]$. Autopsy findings confirmed the platelet-fibrin thrombi in the microvasculature of major organs, including lung, heart, kidney and liver, with or without detectable virions [54]. Hence, immunity-mediated thrombotic events in COVID-19, predominantly in lungs and sporadically in other organs, are closely associated with disease severity and mortality [55].

Similarly, influenza viruses have been previously reported for their detrimental interaction with platelets, manifested as thrombocytopenia, elongated aPTT and elevated D-dimer [56]. Influenza infection stimulates cytokine responses in patients, represented as heightened levels of tumor necrosis factor (TNF)-a and interleukin(IL)-6, activating platelets and forming aggregates with immune cells (e.g., neutrophils and monocytes) [57, 58]. Accrediting to viral pathogenicity, thrombocytopenia and platelet activation depend on influenza virus subtype and SA receptor in the platelets, which induce coagulation disorders to various degrees and lead to thrombotic vascular occlusion [59, 60]. In severe pneumonia induced by either seasonal or pandemic influenza viruses, disseminated intravascular coagulation (DIC) has been found in patients with multiple organ dysfunction syndrome (MODS) and acute respiratory distress syndrome (ARDS), whose anticoagulant (i.e., heparin) treatment lessened severity or improved survival [18].

In contrast, COVID-19 patients demonstrated more intricate thrombotic complications, encompassing low-grade DIC, localized thrombotic microangiopathy (TMA), vein thromboembolism (e.g., deep vein thrombosis) and arterial thrombosis [61]. Notably, COVID19 patients with ARDS displayed much higher platelet hyperreactivity than non-COVID-19 patients with ARDS of equal severity [62]. Compared to the influenza cases, inflammation- and angiogenesis-linked genes that were exclusively altered in COVID-19 patients were found $\sim 40$ times and $>2$ times higher, respectively, suggestive of more profound perivascular inflammation and endothelial injury [63]. As a result, the burden of platelet-rich microthrombi in alveolar capillary of COVID-19 patients was 9 times heavier, and the presence of intussusceptive angiogenesis was $\sim 3$ times more [63]. A clinically important and rare coagulopathy, antiphospholipid autoantibody syndrome was found in COVID-19 patients [64], possibly due to the impaired platelets or/and RBCs turned negatively charged phosphatidylserine of the inner cellular membrane inside out [65], producing antibodies against phospholipids in autoimmune responses. This further triggered platelet activation and thrombin formation, complicating COVID-19-related thrombosis. Recently, a comprehensive comparison on risks of clinical characteristics and mortality patients indicated much higher risks of thrombotic events and death in COVID19 patients than seasonal influenza patients [66], whereas increased risks of thrombosis were highly associated with elevated mortality in COVID-19 patients [67].

Antiviral treatment in seasonal influenza patients with no LMWH administration significantly alleviated thrombocytopenia and most of coagulopathy (except PT), and improved their clinical outcomes. Similarly, after treatment, coagulation dysfunctions including thrombocytopenia, fibrinogen shutdown, and D-dimer elevation in COVID-19 non-ICU patients was significantly improved at the time of hospital discharge; however, despite of LMWH usage in the COVID-19 ICU patients, their coagulopathy remained severe (except mitigated fibrinogen levels), implying persistent thrombosis risks in critically ill patients after hospital discharge. This result was corroborated by others in that 1-3 months after hospital discharge a portion of COVID-19 patients still showed symptoms or were even diagnosed with thrombotic complications, especially for those survived the critical care [68-70]. However, the rate of COVID-19 postdischarge thrombosis is low and non-specific as compared to other acute illness [69].

Finally, yet importantly, our results indicated a higher bleeding incidence during hospitalization in the severe COVID-19 patients who received anticoagulation medication, than either non-severe COVID-19 patients or the influenza patients who did not received anticoagulation. This is supported by other reports where similar frequencies of thrombosis and bleeding in COVID-19 patients were observed, comparable to the situations in other critical illness of similar severity, and this in-hospital bleeding might be associated with the intensified anticoagulant treatment [71-73]. Moreover, the hemorrhagic rate was also found close to that of thrombosis after months following hospital discharge $[68,70]$.

The study has some limitations. First, our conclusion might be limited by patient number and case availability. Although 461 patients included in this study might be representative of COVID-19 cases in Hubei province 
during the early outbreak in China, the disease development and the patient outcome could be changing as management and treatment of COVID-19 are quickly evolving worldwide. For 409 seasonal influenza patients, given the fact that the ratio of severe patients in general population is low, especially in a non-epidemic period, only mild to moderate patients who did not require critical care are available and included in this study. Second, due to the emergency of COVID19 as a major infectious disease, many laboratory tests were unavailable on spot, such as ultrasonography to diagnose possible pulmonary embolism in critically ill patients, and some critical parameters in monitoring hematological changes of patients over time were absent, such as coagulation and cytokine profiles. This would otherwise elucidate more about the coagulopathy of this deadly viral disease.

Put together, our results point out that the platelet injury by SARS-CoV-2 infection with consequence of coagulopathy and thrombi formation in severe COVID19 patients may take responsibility for the disease fatality and sustain a longstanding prothrombotic state in COVID-19 survivors. Compared to influenza cases, platelet hyperreactivity in COVID-19 is more evident, associated with worse hyper-inflammatory response and more refractory coagulopathy. Early antibiotic and antiviral interventions in COVID-19 patients could efficiently attenuate the coagulation disorders, and thromboprophylaxis together with immunomodulatory medications (such as inflammation reducers) in severe patients may help dampen the platelet reactivity, so lessening the severity and lowering the mortality. However, as bleeding complications occur in hospitalized COVID-19 patients and may continue after hospital discharge, extended thromboprophylaxis for COVID-19 survivors should be carefully evaluated.

\section{Supplementary Information}

The online version contains supplementary material available at https://doi. org/10.1186/s40164-021-00228-z.

\footnotetext{
Additional file 1: Table S1. Baseline characteristics, blood parameters and coagulation factors in the non-ICU and the ICU groups of COVID19 cohorts, compared to those in the influenza cohort, respectively. Table S2. Comparison of blood parameters within the COVID-19 cohort between the non-ICU group and the ICU survivors before and after treatment, respectively. Table S3. Comparison of bleeding events, usage of hemostatic drugs or component transfusion within the COVID-19 cohort between the non-ICU and the ICU groups, between survivors and non-survivors in the ICU groups, respectively. In addition, the non-ICU or the ICU groups of COVID-19 cohort was also compared to the influenza cohort, respectively. Figure S1. Frequency of each blood cell count and coagulation character was plotted between (A) COVID-19 cohort versus influenza cohort; (B) ICU group versus non-ICU group within the COVID19 cohort; (C) non-survivors versus survivors within the COVID-19 ICU group. Diagonal line (dotted) indicated a hypothetically equal frequency between two groups.
}

Acknowledgements

We thank the financial support from Jiangsu University.

\section{Authors' contributions}

J.Z. and Z.T. conceived the idea and designed the study. J.Z., X.H., D.D., and Z.T. contributed to the data acquisition, processing, and table/figure preparation. J.Z., X.H., D.D., and Z.T. contributed to the manuscript writing. J.Z., X.H., and Z.T. contributed to the statistical analysis. All authors reviewed, read and approved the final manuscript.

\section{Funding \\ None.}

Availability of data and materials

Data available on request due to privacy/ethical restrictions.

\section{Declarations}

\section{Ethics approval and consent to participate}

This study was approved by the First People's Hospital of Jiangxia District (FPHJD) at Wuhan and the Huangshi City Hospital $(\mathrm{HCH})$ of Hubei Province, and by the Affiliated Hospital of Jiangsu University (AHJU) in Zhenjiang City of Jiangsu Province, in China, respectively. Patient information remains anonymous, and written consent was waived by Ethics Commission of FPHJD, HCH and $\mathrm{AHJU}$, respectively.

\section{Consent for publication}

Not applicable.

\section{Competing interests}

The authors declared no competing interests.

\section{Author details \\ ${ }^{1}$ Jiangsu Province Key Laboratory of Medical Science and Laboratory Medi- cine, School of Medicine, Jiangsu University, Zhenjiang 212013, Jiangsu, China. ${ }^{2}$ Department of Critical Care Medicine, The Affiliated Hospital, Jiangsu Univer- sity, Zhenjiang 212001, Jiangsu, China. ${ }^{3}$ Center for Evidence-Based and Trans- lational Medicine, Zhongnan Hospital of Wuhan University, Wuhan 430071, China. ${ }^{4}$ Department of Critical Care Medicine, The First People's Hospital of Jiangxia District, Wuhan 430200, Hubei, China.}

Received: 23 March 2021 Accepted: 24 May 2021

Published online: 31 May 2021

References

1. Organization WH. COVID-19 weekly epidemiological update, 2021.

2. Guan WJ, Ni ZY, Hu Y, Liang WH, Ou CQ, He JX, Liu L, Shan H, Lei CL, Hui DSC, et al. Clinical characteristics of coronavirus disease 2019 in China. N Engl J Med. 2020;382(18):1708-20.

3. Zhu N, Zhang D, Wang W, Li X, Yang B, Song J, Zhao X, Huang B, Shi W, $L u R$, et al. A novel coronavirus from patients with pneumonia in China, 2019. N Engl J Med. 2020;382(8):727-33.

4. Zhou P, Yang XL, Wang XG, Hu B, Zhang L, Zhang W, Si HR, Zhu Y, Li B, Huang $C L$, et al. A pneumonia outbreak associated with a new coronavirus of probable bat origin. Nature. 2020;579(7798):270-3.

5. Hoffmann M, Kleine-Weber H, Schroeder S, Kruger N, Herrler T, Erichsen S, Schiergens TS, Herrler G, Wu NH, Nitsche A, et al. SARS-CoV-2 cell entry depends on ACE2 and TMPRSS2 and is blocked by a clinically proven protease inhibitor. Cell. 2020;181(2):271-280 e278.

6. Zou X, Chen K, Zou J, Han P, Hao J, Han Z. Single-cell RNA-seq data analysis on the receptor ACE2 expression reveals the potential risk of different human organs vulnerable to 2019-nCoV infection. Front Med. 2020;14(2):185-92.

7. Wang W, Xu Y, Gao R, Lu R, Han K, Wu G, Tan W. Detection of SARS-CoV-2 in different types of clinical specimens. JAMA. 2020;323(18):1843-4.

8. Richardson S, Hirsch JS, Narasimhan M, Crawford JM, McGinn T, Davidson KW, and the Northwell C-RC, Barnaby DP, Becker LB, Chelico JD, et al. Presenting characteristics, comorbidities, and outcomes among 5700 
patients hospitalized with COVID-19 in the New York City area. JAMA. 2020; 323(20):2052-9

9. Wang D, Hu B, Hu C, Zhu F, Liu X, Zhang J, Wang B, Xiang H, Cheng Z, Xiong Y, et al. Clinical characteristics of 138 hospitalized patients with 2019 novel coronavirus-infected pneumonia in Wuhan, China. JAMA. 2020;323(11):1061-9.

10. Huang C, Wang Y, Li X, Ren L, Zhao J, Hu Y, Zhang L, Fan G, Xu J, Gu X, et al. Clinical features of patients infected with 2019 novel coronavirus in Wuhan, China. Lancet. 2020:395(10223):497-506.

11. Tang N, Li D, Wang X, Sun Z. Abnormal coagulation parameters are associated with poor prognosis in patients with novel coronavirus pneumonia. J Thromb Haemost. 2020;18(4):844-7.

12. Tang N, Bai H, Chen X, Gong J, Li D, Sun Z. Anticoagulant treatment is associated with decreased mortality in severe coronavirus disease 2019 patients with coagulopathy. J Thromb Haemost. 2020;18(5):1094-9.

13. Wichmann D, Sperhake JP, Lutgehetmann M, Steurer S, Edler C, Heinemann A, Heinrich F, Mushumba H, Kniep I, Schroder AS, et al. Autopsy findings and venous thromboembolism in patients with COVID-19: a prospective cohort study. Ann Intern Med. 2020;173(4):268-77.

14. Lax SF, Skok K, Zechner P, Kessler HH, Kaufmann N, Koelblinger C, Vander K, Bargfrieder U, Trauner M. Pulmonary arterial thrombosis in COVID-19 with fatal outcome : results from a prospective, single-center, clinicopathologic case series. Ann Intern Med. 2020;173(5):350-61.

15. Hanley B, Naresh KN, Roufosse C, Nicholson AG, Weir J, Cooke GS, Thursz M, Manousou P, Corbett R, Goldin R, et al. Histopathological findings and viral tropism in UK patients with severe fatal COVID-19: a post-mortem study. Lancet Microbe. 2020;1(6):e245-53.

16. Fox SE, Akmatbekov A, Harbert JL, Li G, Quincy Brown J, Vander Heide RS. Pulmonary and cardiac pathology in African American patients with COVID-19: an autopsy series from New Orleans. Lancet Respir Med. 2020;8(7):681-6.

17. van Gorp EC, Suharti C, ten Cate H, Dolmans WM, van der Meer JW, ten Cate JW, Brandjes DP. Review: infectious diseases and coagulation disorders. J Infect Dis. 1999;180(1):176-86.

18. Yang $Y$, Tang H. Aberrant coagulation causes a hyper-inflammatory response in severe influenza pneumonia. Cell Mol Immunol. 2016;13(4):432-42.

19. Zhang J, Ding D, Huang $X$, Zhang J, Chen D, Fu P, Shi Y, Xu W, Tao Z. Differentiation of COVID-19 from seasonal influenza: a multicenter comparative study. J Med Virol. 2021;93(3):1512-9.

20. LiT. Diagnosis and clinical management of severe acute respiratory syndrome Coronavirus 2 (SARS-CoV-2) infection: an operational recommendation of Peking Union Medical College Hospital (V2.0). Emerg Microbes Infect. 2020;9(1):582-5.

21. Harrison AG, Lin T, Wang P. Mechanisms of SARS-CoV-2 transmission and pathogenesis. Trends Immunol. 2020;41(12):1100-15.

22. Petrova VN, Russell CA. The evolution of seasonal influenza viruses. Nat Rev Microbiol. 2018;16(1):60.

23. Krammer F, Smith GJD, Fouchier RAM, Peiris M, Kedzierska K, Doherty PC, Palese P, Shaw ML, Treanor J, Webster RG, et al. Influenza. Nat Rev Dis Primers. 2018;4(1):3

24. Sungnak W, Huang N, Becavin C, Berg M, Queen R, Litvinukova M, Talavera-Lopez C, Maatz H, Reichart D, Sampaziotis F, et al. SARS-CoV-2 entry factors are highly expressed in nasal epithelial cells together with innate immune genes. Nat Med. 2020;26(5):681-7.

25. Bunyavanich S, Do A, Vicencio A. Nasal gene expression of angiotensinconverting enzyme 2 in children and adults. JAMA. 2020;323(23):2427-9.

26. Morniroli D, Gianni ML, Consales A, Pietrasanta C, Mosca F. Human sialome and coronavirus disease-2019 (COVID-19) pandemic: an understated correlation? Front Immunol. 2020;11:1480

27. Tortorici MA, Walls AC, Lang Y, Wang C, Li Z, Koerhuis D, Boons GJ, Bosch BJ, Rey FA, de Groot RJ, et al. Structural basis for human coronavirus attachment to sialic acid receptors. Nat Struct Mol Biol. 2019;26(6):481-9.

28. Baker AN, Richards SJ, Guy CS, Congdon TR, Hasan M, Zwetsloot AJ, Gallo A, Lewandowski JR, Stansfeld PJ, Straube A, et al. The SARS-COV-2 spike protein binds sialic acids and enables rapid detection in a lateral flow point of care diagnostic device. ACS Cent Sci. 2020;6(11):2046-52.

29. Yang J, Petitjean SJL, Koehler M, Zhang Q, Dumitru AC, Chen W, Derclaye S, Vincent SP, Soumillion P, Alsteens D. Molecular interaction and inhibition of SARS-CoV-2 binding to the ACE2 receptor. Nat Commun. 2020;11(1):4541.
30. Bai L, Zhao Y, Dong J, Liang S, Guo M, Liu X, Wang X, Huang Z, Sun X, Zhang Z, et al. Coinfection with influenza A virus enhances SARS-CoV-2 infectivity. Cell Res. 2021. https://doi.org/10.1038/s41422-021-00473-1.

31. Piroth L, Cottenet J, Mariet AS, Bonniaud P, Blot M, Tubert-Bitter P, Quantin C. Comparison of the characteristics, morbidity, and mortality of COVID19 and seasonal influenza: a nationwide, population-based retrospective cohort study. Lancet Respir Med. 2020. https://doi.org/10.1016/S22132600(20)30527-0.

32. Song X, Delaney M, Shah RK, Campos JM, Wessel DL, DeBiasi RL. Comparison of clinical features of COVID-19 vs seasonal influenza A and B in US children. JAMA Netw Open. 2020;3(9):e2020495.

33. Dehingia N, Raj A. Sex differences in COVID-19 case fatality: do we know enough? Lancet Glob Health. 2021;9(1):e14-5.

34. Peckham H, de Gruijter NM, Raine C, Radziszewska A, Ciurtin C, Wedderburn LR, Rosser EC, Webb K, Deakin CT. Male sex identified by global COVID-19 meta-analysis as a risk factor for death and ITU admission. Nat Commun. 2020;11(1):6317.

35. Luo Y, Yuan X, Xue Y, Mao L, Lin Q, Tang G, Song H, Liu W, Hou H, Wang F. Using a diagnostic model based on routine laboratory tests to distinguish patients infected with SARS-CoV-2 from those infected with influenza virus. Int J Infect Dis. 2020;95:436-40.

36. Liu X, Zhang R, He G. Hematological findings in coronavirus disease 2019: indications of progression of disease. Ann Hematol. 2020;99(7):1421-8.

37. Izcovich A, Ragusa MA, Tortosa F, Lavena Marzio MA, Agnoletti C, Bengolea A, Ceirano A, Espinosa F, Saavedra E, Sanguine V, et al. Prognostic factors for severity and mortality in patients infected with COVID-19: a systematic review. PLoS ONE. 2020;15(11):e0241955.

38. Tao Z, Xu J, Chen W, Yang Z, Xu X, Liu L, Chen R, Xie J, Liu M, Wu J. Anemia is associated with severe illness in COVID-19: a retrospective cohort study. J Med Virol. 2021;93(3):1478-88.

39. Bergamaschi G, de Andreis FB, Aronico N, Lenti MV, Barteselli C, Merli S, Pellegrino I, Coppola L, Cremonte EM, Croce G, et al. Anemia in patients with COVID-19: pathogenesis and clinical significance. Clin Exp Med. 2021. https://doi.org/10.1007/s10238-020-00679-4.

40. Wang K, Chen W, Zhang Z, Deng Y, Lian JQ, Du P, Wei D, Zhang Y, Sun XX, Gong $L$, et al. CD147-spike protein is a novel route for SARS-CoV-2 infection to host cells. Signal Transduct Target Ther. 2020;5(1):283.

41. Mehta P, Haskard DO, Laffan MA, Chambers RC, Hunt BJ. Thromboses and COVID-19: reducing inflammation in addition to thromboprophylaxis. Lancet Rheumatol. 2021;3(3):e171-2.

42. Reade MC, Weissfeld L, Angus DC, Kellum JA, Milbrandt EB. The prevalence of anemia and its association with 90-day mortality in hospitalized community-acquired pneumonia. BMC Pulm Med. 2010;10:15.

43. Rauff B, Idrees M, Shah SA, Butt S, Butt AM, Ali L, Hussain A, Irshad Ur R, Ali M. Hepatitis associated aplastic anemia: a review. Virol J. 2011;8:87.

44. Mei Y, Weinberg SE, Zhao L, Frink A, Qi C, Behdad A, Ji P. Risk stratification of hospitalized COVID-19 patients through comparative studies of laboratory results with influenza. EClinicalMedicine. 2020;26:100475.

45. Mudd PA, Crawford JC, Turner JS, Souquette A, Reynolds D, Bender D, Bosanquet JP, Anand NJ, Striker DA, Martin RS, et al. Distinct inflammatory profiles distinguish COVID-19 from influenza with limited contributions from cytokine storm. Sci Adv. 2020. https://doi.org/10.1126/sciadv.abe30 24.

46. Liao D, Zhou F, Luo L, Xu M, Wang H, Xia J, Gao Y, Cai L, Wang Z, Yin P, et al. Haematological characteristics and risk factors in the classification and prognosis evaluation of COVID-19: a retrospective cohort study. Lancet Haematol. 2020;7(9):e671-8.

47. Lefrançais E, Ortiz-Muñoz G, Caudrillier A, Mallavia B, Liu F, Sayah DM, Thornton EE, Headley MB, David T, Coughlin SR. The lung is a site of platelet biogenesis and a reservoir for haematopoietic progenitors. Nature. 2017;544(7648):105-9.

48. Zhang S, Liu Y, Wang X, Yang L, Li H, Wang Y, Liu M, Zhao X, Xie Y, Yang Y, et al. SARS-CoV-2 binds platelet ACE2 to enhance thrombosis in COVID19. J Hematol Oncol. 2020:13(1):120

49. Manne BK, Denorme F, Middleton EA, Portier I, Rowley JW, Stubben C, Petrey AC, Tolley ND, Guo L, Cody M, et al. Platelet gene expression and function in patients with COVID-19. Blood. 2020;136(11):1317-29.

50. Levi M, Thachil J, Iba T, Levy JH. Coagulation abnormalities and thrombosis in patients with COVID-19. Lancet Haematol. 2020;7(6):e438-40.

51. Wright FL, Vogler TO, Moore EE, Moore HB, Wohlauer MV, Urban S, Nydam TL, Moore PK, McIntyre RC Jr. Fibrinolysis shutdown correlation with 
thromboembolic events in severe COVID-19 infection. J Am Coll Surg. 2020;231(2):193-203 e191.

52. Middleton EA, He XY, Denorme F, Campbell RA, Ng D, Salvatore SP, Mostyka M, Baxter-Stoltzfus A, Borczuk AC, Loda M, et al. Neutrophil extracellular traps contribute to immunothrombosis in COVID-19 acute respiratory distress syndrome. Blood. 2020;136(10):1169-79.

53. Hottz ED, Azevedo-Quintanilha IG, Palhinha L, Teixeira L, Barreto EA, Pao CRR, Righy C, Franco S, Souza TML, Kurtz P, et al. Platelet activation and platelet-monocyte aggregate formation trigger tissue factor expression in patients with severe COVID-19. Blood. 2020;136(11):1330-41.

54. Rapkiewicz AV, Mai X, Carsons SE, Pittaluga S, Kleiner DE, Berger JS, Thomas S, Adler NM, Charytan DM, Gasmi B, et al. Megakaryocytes and platelet-fibrin thrombi characterize multi-organ thrombosis at autopsy in COVID-19: a case series. EClinicalMedicine. 2020;24:100434.

55. Loo J, Spittle DA, Newnham M. COVID-19, immunothrombosis and venous thromboembolism: biological mechanisms. Thorax. 2021. https:// doi.org/10.1136/thoraxjnl-2020-216243.

56. Popa E, Rusu-Cordunean F. Platelets and influenza A (N1H1). Eur Respir J. 2011;38(Suppl 55):p4349.

57. Boilard E, Pare G, Rousseau M, Cloutier N, Dubuc I, Levesque T, Borgeat P, Flamand L. Influenza virus H1N1 activates platelets through FcgammaRIIA signaling and thrombin generation. Blood. 2014;123(18):2854-63.

58. Rondina MT, Brewster B, Grissom CK, Zimmerman GA, Kastendieck DH, Harris ES, Weyrich AS. In vivo platelet activation in critically ill patients with primary 2009 influenza A(H1N1). Chest. 2012;141(6):1490-5.

59. Jansen AG, Spaan T, Low HZ, Di lorio D, Van Den Brand J, Tieke M, Barendrecht A, Rohn K, Van Amerongen G, Stittelaar K. Influenzainduced thrombocytopenia is dependent on the subtype and sialoglycan receptor and increases with virus pathogenicity. Blood Adv. 2020;4(13):2967-78.

60. Koupenova M, Corkrey HA, Vitseva O, Manni G, Pang CJ, Clancy L, Yao C, Rade J, Levy D, Wang JP, et al. The role of platelets in mediating a response to human influenza infection. Nat Commun. 2019;10(1):1780.

61. Iba T, Levy JH, Connors JM, Warkentin TE, Thachil J, Levi M. The unique characteristics of COVID-19 coagulopathy. Crit Care. 2020;24(1):360

62. Zaid Y, Guessous F, Puhm F, Elhamdani W, Chentoufi L, Morris AC, Cheikh A, Jalali F, Boilard E, Flamand L. Platelet reactivity to thrombin differs between patients with COVID-19 and those with ARDS unrelated to COVID-19. Blood Adv. 2021;5(3):635-9.

63. Ackermann M, Verleden SE, Kuehnel M, Haverich A, Welte T, Laenger F, Vanstapel A, Werlein C, Stark H, Tzankov A, et al. Pulmonary vascular endothelialitis, thrombosis, and angiogenesis in COVID-19. N Engl J Med. 2020;383(2):120-8.

64. Zhang Y, Xiao M, Zhang S, Xia P, Cao W, Jiang W, Chen H, Ding X, Zhao $H$, Zhang $H$, et al. Coagulopathy and antiphospholipid antibodies in patients with COVID-19. N Engl J Med. 2020;382(17):e38.

65. Weisel JW, Litvinov RI. Red blood cells: the forgotten player in hemostasis and thrombosis. J Thromb Haemost. 2019:17(2):271-82.

66. Xie Y, Bowe B, Maddukuri G, Al-Aly Z. Comparative evaluation of clinical manifestations and risk of death in patients admitted to hospital with COVID-19 and seasonal influenza: cohort study. BMJ. 2020;371:m4677.

67. Malas MB, Naazie IN, Elsayed N, Mathlouthi A, Marmor R, Clary B. Thromboembolism risk of COVID-19 is high and associated with a higher risk of mortality: a systematic review and meta-analysis. EClinicalMedicine. 2020;29:100639.

68. Patell R, Bogue T, Koshy A, Bindal P, Merrill M, Aird WC, Bauer KA, Zwicker Jl. Postdischarge thrombosis and hemorrhage in patients with COVID-19. Blood. 2020;136(11):1342-6.

69. Roberts LN, Whyte MB, Georgiou L, Giron G, Czuprynska J, Rea C, Vadher B, Patel RK, Gee E, Arya R. Postdischarge venous thromboembolism following hospital admission with COVID-19. Blood. 2020;136(11):1347-50.

70. Whyte MB, Barker R, Kelly PA, Gonzalez E, Czuprynska J, Patel RK, Rea C, Perrin F, Waller M, Jolley C, et al. Three-month follow-up of pulmonary embolism in patients with COVID-19. Thromb Res. 2021;201:113-5.

71. Jiménez D, García-Sanchez A, Rali P, Muriel A, Bikdeli B, Ruiz-Artacho P, Le Mao R, Rodríguez C, Hunt BJ, Monreal M. Incidence of venous thromboembolism and bleeding among hospitalized patients with COVID-19: a systematic review and meta-analysis. Chest. 2020.

72. Al-Samkari H, Gupta S, Leaf RK, Wang W, Rosovsky RP, Brenner SK, Hayek SS, Berlin H, Kapoor R, Shaefi S, et al. Thrombosis, bleeding, and the observational effect of early therapeutic anticoagulation on survival in critically ill patients with COVID-19. Ann Intern Med. 2021.

73. Al-Samkari H, Karp Leaf RS, Dzik WH, Carlson JCT, Fogerty AE, Waheed A, Goodarzi K, Bendapudi PK, Bornikova L, Gupta S, et al. COVID-19 and coagulation: bleeding and thrombotic manifestations of SARS-CoV-2 infection. Blood. 2020;136(4):489-500.

\section{Publisher's Note}

Springer Nature remains neutral with regard to jurisdictional claims in published maps and institutional affiliations.
Ready to submit your research? Choose BMC and benefit from:

- fast, convenient online submission

- thorough peer review by experienced researchers in your field

- rapid publication on acceptance

- support for research data, including large and complex data types

- gold Open Access which fosters wider collaboration and increased citations

- maximum visibility for your research: over $100 \mathrm{M}$ website views per year

At BMC, research is always in progress.

Learn more biomedcentral.com/submissions 